polyarthritis) showed a decrease in the content of the main fractions of polar phospholipids and an increase in the phospholipid lysoforms and free fatty acids by $8.9 \% 21.4 \%$ and $9 \%$ compared with the control group. This leads to an increase erythrocytes membranes microviscosity. Dyslipidaemia was combined with body composition changes. In this patients IMCT was higher than in control group $(0.73+0.03$ versus $0.45+0.02, \mathrm{p}<0.05)$.

In high disease activity both systemic onset and polyarticular JIA proatherogenic lipid disturbances and vascular disorders took place.

\section{HENOCH-SCHONLEIN PURPURA IN PEDIATRICS: FIVE YEARS OF EXPERIENCE AT A RESEARCH AND TRAINING HOSPITAL IN ISTANBUL}

Burcu Karadas*, Zehra Esra Onal. Haydarpasa Research and Training Hospital/Istanbul

10.1136/archdischild-2021-europaediatrics.437

Our aim was to describe the epidemiological, clinical, laboratory characteristics of patients with HSP and to compare them existing studies.

The medical records of children with HSP, who met the Ankara criteria for HSP diagnosis, were retrospectively reviewed at the Haydarpaşa Research and Training Hospital between the years 2010-2016. The epidemiological, etiological and clinical features, laboratory results were assessed.

In our study, the age of the patients ranged from 3 to 12 years (mean age 7 years). 93.3\% of the patients were under the age of $10 .(53.3 \%)$ were male and 21 (46.6\%) were female. The male/female ratio was $1.14 / 1$.

In our study, 24 cases $(53.3 \%)$ were most seen in the winter season, followed by the autumn season with 12 cases (26.67\%), there was significant clustering in the winter-autumn seasons.

In our study, 21 cases (46.67\%) had symptoms of upper respiratory infection in the last few weeks, 9 (20\%) had analgesic- antipyretic and/or antibiotics use.6 cases (13.3\%) described as acute gastroenteritis and/or diarrhea, and 9 patients (20\%) had a high fever. In 10 (22.2\%) patients, no etiological factors could be identified.

Clinical features examined; 44 (97.7\%) of patients applied to the health institution with purpura, $22(48.8 \%)$ joint pain and/or swelling, $10(22.2 \%)$ abdominal pain and $5(11.1 \%)$ with fever. Only 1 patient $(2.2 \%)$ complained at the time of application that they could not only walk without rash.

Anemia was detected in $6(13.3 \%)$ patients and leukocytosis in $30(66.6 \%)$ patients. Trombocytosis was found in 24 $(53.3 \%)$ patients. Erythrocyte sedimentation rate was high in $66 \%$ of patients, while CRP was positive in $73.3 \%$ of patients. Microscopic hematuria was found in 14 (31.1\%) patients, proteinuria was found in $6(13.3 \%)$ patients.Total IgA value was high in 5 patients (11\%), C3 and C4 were found to be normal in all. PT/INR values were normal in all patients.

Our results are compatible with the literature, there are no significant differences in the epidemiological and clinical profile than reported elsewhere except IgA values in the studies conducted in the literature are significantly higher than in our study.

\section{RELEVANCE OF ADALIMUMAB SERUM CONCENTRATION AND ANTI-ADALIMUMAB ANTIBODIES IN SECONDARY LOSS OF RESPONSE IN PATIENTS WITH JUVENILE IDIOPATHIC ARTHRITIS}

Nikolina Benco Kordić*, Ana Kozmar, Alenka Gagro. Children's Hospital Zagreb, Croatia

10.1136/archdischild-2021-europaediatrics.438

Adalimumab (ADA) is fully humanized, monoclonal antibody against tumour necrosis factor (TNF- $\alpha$ ), and is efficacious biological treatment option for patients with juvenile idiopathic arthritis (JIA). However, some patients who respond to the ADA therapy subsequently lose response to this treatment and present with relapse of JIA.

In a period of five years (2015-2020), a cohort of 19 patients with JIA, treated with ADA in Children's Hospital Zagreb, was followed. Disease activity was evaluated based on counts of joints with active disease, erythrocyte sedimentation rate (ESR) and physician global assessment of disease activity. Blood samples were collected from patients who presented with JIA relapse and suspected loss of response (LOR), as well as randomly from patients who had achieved appropriate disease control with ADA therapy as a control group. Levels of adalimumab and anti-adalimumab antibodies (AAA) were measured using enzyme-linked immunosorbent assays (ELISA) (Immundiagnostik AG, Bensheim, Germany). Use of concomitant methotrexate (MTX) therapy was noted, as well as time of AAA development from the beginning of ADA therapy.

Out of 19 patients on ADA, six presented with LOR. Out of six, positive AAA and subtherapeutic ADA concentrations were found in four patients, while in two patients ADA concentrations were subtherapeutic but AAA negative. Two of the six relapsed patients did not receive MTX at the time of serum sampling due to adverse reactions to this drug. The median time to development of relapse of JIA and proven AAA was eleven months (2-20 months) after initiation of ADA therapy. Adalimumab has so far been replaced by tocilizumab in four patients with LOR. Of the thirteen patients in the control group, two had positive AAA and subtherapy ADA concentrations, and one had only subtherapy ADA concentrations. Four of the thirteen patients in the control group did not receive MTX at the time of sampling, all four had negative AAA, and one also had a subtherapeutic concentration of ADA.

In our patients with relapsed JIA, positive AAA and subtherapeutic ADA concentrations were associated with loss of therapeutic response. However, the occurrence of AAA and subtherapeutic concentrations of ADA in the control group also indicate the need for further research in a larger number of patients, as well as the need for serial measurement and testing the ability of AAA to neutralize ADA.

\section{NEW CANDIDATE BIOMARKER OF NEPHRITIS RISK ASSESSMENT IN CHILDREN WITH HENOCH-SCHÖNLEIN PURPURA}

Marijan Frković*, Ana Mlinarić, Saša Sršen, Alenka Gagro, Dunja Rogić, Marija Jelušić. Department of Paediatrics, University Hospital Center Zagreb, University of Zagreb School of Medicine

10.1136/archdischild-2021-europaediatrics.439 
Goal Henoch-Schönlein purpura (HSP) is the most common vasculitis of the childhood. Among all possible symptoms/ complications, nephritis (HSPN) is the main and almost only cause of morbidity and mortality in HSP. The aim of the study was to investigate the value of erythrocyte glutathione S-transferase (e-GST) activity as an early predictor of HSPN.

Methods Ninety-seven children with HSP were enrolled into the study. The control group consisted of 52 children without clinical and laboratory signs of inflammation. In all participants e-GST activity was determined spectrometrically from the whole blood samples, after incubation with a commercial GST assay.

Results At the beginning of the disease the e-GST activity was significantly higher in HSPN patients who developed the nephritis during the six month follow up period, compared to the group of HSP patients without signs of nephritis: median (interquartile range) $5,70 \mathrm{U} / \mathrm{mgHb} \quad(4,38-7,50 \mathrm{U} /$ $\mathrm{mgHb})$ compared to $3,10 \mathrm{U} / \mathrm{mgHb}(2,20-4,20 \mathrm{U} / \mathrm{mgHb})$; $\mathrm{P}<0,001$. Similar results were obtained after the comparison of the HSPN patients and control group: $5,70 \mathrm{U} / \mathrm{mgHb}$ $(4,38-7,50 \mathrm{U} / \mathrm{mgHb})$ vs.3,13 U/mgHb (1,91-4,20 U/mgHb); $\mathrm{P}<0,001$. There were no statistically significant differences between the group of HSP patients without nephritis and control group $(\mathrm{P}=0,837)$. During the follow up period, a significant decrease of e-GST activity was observed in the HSPN patients, but it was still significantly higher compared to the group of HSP patients without nephritis $(\mathrm{P}<0,001 /$ $\mathrm{P}<0,001)$.

Conclusion e-GST activity is a reliable, independent marker of early nephritis risk assessment in children with HSP. As a sensitive, specific and feasible laboratory test, it has potential practical utility in the diagnostic algorithm and monitoring of the children with HSP.

\section{SAPHO SYNDROME IN CHILDHOOD}

Helena Munivrana Škvorc*, Marko Škvorc, Marija Šenjug Perica, Lana Tambić Bukovac. Children's Hospital Srebrnjak

\subsection{6/archdischild-2021-europaediatrics.440}

SAPHO syndrome is an acronym for 'Synovitis, Acne, Pustulosis, Hyperostosis and Osteitis'. It is combination of cutaneous and musculoskeletal manifestations, such as osteoarthritis and hyperostosis of bones of the anterior chest wall associated with acne fulminans and hidradenitis suppurativa. We present the case of a 15-year old boy with osteoarthritis of the left hip, osteitis of the left carpal bones and right clavicula with acne conglobata. Bone biopsy of the left hip has been carried out and Propionibacterium acnes was found in the bone lesion.

Identification of the Propionibacterium acnes from bone biopsy in SAPHO syndrome has occasionally been reported, which could suggest that microorganisms could be a trigger for osteitis and hyperostosis and may play the role in the pathogenesis of the disease. Our patient was treated with nonsteroidal anti-inflammatory drugs, antibiotics and a corticosteroids, which resulted in clinical improvement, but not complete remission, so we introduced methotrexate in therapy.
The purpose of this case study is to raise awareness to a set of clinical features of SAPHO syndrome and its early recognition and prompt therapy.

\section{ASSOCIATION BETWEEN GASTROINTESTINAL MANIFESTATIONS AND THE RISK OF RENAL DISEASE IN CHILDREN WITH IGA VASCULITIS}

Mario Sestan*, Nastasia Kifer, Sasa Srsen, Aleksandar Ovuka, Mateja Batnozic Varga, Matej Sapina, Martina Held, Maja Ban, Ana Kozmar, Marijana Coric, Stela Bulimbasic, Kristina Crkvenac, Danko Milosevic, Marijan Frkovic, Alenka Gagro, Marija Jelusic. University Hospital Centre Zagreb, Department of Paediatrics, University of Zagreb School of Medicine

\subsection{6/archdischild-2021-europaediatrics.441}

IgA vasculitis (IgAV) is the most common childhood-vasculitis in which more than $50 \%$ of children develop gastrointestinal (GI) symptoms. In $10-20 \%$ of patients serious complications such as intussusception, bowel perforation, and massive bleeding may occur. The most important complication is the development of nephritis with progression to chronic renal failure in about $3 \%$ of children. The aim of the research was to analyze clinical and biochemical parameters in patients with IgAV and GI manifestations.

This retrospective study included children with IgAV reviewed in five Croatian University Centers for pediatric rheumatology in the period 2009 to 2019.

Out of 611 children with IgAV, 320 were males and 291 were females. The overall GI symptoms prevalence was $45.9 \%$ and the median (range) age at diagnosis was 6.42 (4.5-8.83) years. Among patients with GI symptoms there were 1.44 times more males $(\mathrm{N}=166)$ than females $(\mathrm{N}=115)$, which was statistically significant $(p=0.003)$. Patients with GI symptoms had less infections before the appearance of purpura $(59.8 \%$ vs. $70.9 \%, p=0.005)$ and were found to be significantly more likely to have rash distributed on the trunk $(61.9 \%$ vs. $48.5 \%, p=0.001)$, and upper extremities (35.2\% vs. $24.7 \%$, $\mathrm{p}=0.006)$, as well as generalized rash $(38.8 \%$ vs. $28.3 \%$, $\mathrm{p}=0.008)$. These patients also had significantly higher values of C-reactive protein, leukocyte count, erythrocytes and platelets, hemoglobin, hematocrit and D-dimer concentrations and lower levels of $\operatorname{IgG}$ and IgM. In our cohort 42 out of 281 children (14.9\%) had the most severe GI manifestations (intussusception and/or massive GI bleeding) with significantly higher values of 24-hour urine protein levels and D-dimer concentrations and lower total serum protein, albumin, IgG, IgM and C3 levels in comparison with children whose GI manifestations were not severe. Predictors of severe GI involvement were: relapse of the disease, generalized rash, rash extended on upper extremities, rash extended to the face, recurrent rash and renal involvement, as well as lower values of prothrombin time, fibrinogen and $\operatorname{IgM}$ among the laboratory parameters. Patients with GI symptoms were 1.68 times more likely to develop nephritis, and this probability was 2.58 times higher if GI symptoms occurred before other symptoms. Other predictors of nephritis were: severe and moderate GI manifestations, recurrent rash, one or more relapses of IgAV, and older age.

Older children with IgAV and severe GI manifestations in whom IgAV begun with GI symptoms had a higher risk of acute and chronic complications of the disease.

SUPPORT: Croatian Science Foundation project IP-2019-048822 